\title{
Titanium oxide based nanotubes, nanofibers, nanoflowers, and nanodiscs
}

Huifang $\mathrm{Xu}^{*}$, Yingbing Jiang*, Zimin Nie*, Jinhuy Lee**, Hiromi Konishi*, and Yifeng Wang***, and Marek Osinski**

*Department of Earth and Planetary Sciences, University of New Mexico, Albuquerque, NM 97109

**Center for High Technology Materials, University of New Mexico, Albuquerque, NM 87131

***Sandia National Laboratories, Carlsbad, NM 88220

Nanomaterials are of current interest due to their unique properties and potential applications in catalysis and optoelectronic devices. Inherent crystallographic structures of materials play crucial roles in both physical and chemical properties. For instance, structures and properties appear different between cubic sphalerite $\mathrm{ZnS}$ and hexagonal wurtzite one. Both structure and size controls of nanocrystals are quite important in preparative chemistry and materials sciences. The shape control of nanosized crystals is another important factor for an as-prepared product. The elongate one-dimensional (1-D) morphology such as nanorods (nanowires, nanofibers, nanowhiskers), nanobelts, and nanotubes has been focused extensively due to their specific density of electronic state and wide applications.

Titanium oxides of $\mathrm{TiO}_{2}$ and titanates are interesting materials because of their semiconducting properties. Synthesis of shape and texture controlled materials will have potential applications in optoelectronics and photocatalysis. We have synthesized titanium oxide based nanotubes with different tube diameters (Figure 1). We also synthesized titanium oxide based nanofibers, nano flowers, and nanodisc (Figures $2-4$ ). Our preliminary data indicate that solution condition, reaction temperature, and staring $\mathrm{Ti}$ materials affect the formation and morphologies of the titanate phases. High temperature favors the formation of nanofibers. However, low temperature favors the formation of plate-like shapes. TiN substrate will lead to the formation of nanoflowers and nanodiscs (Figures 3 and 4). Long nanotubes can be synthesized by doing certain impurities into the structure. Photoluminescence $(\mathrm{PL})$ of the nanotubes with different tube diameters give different PL peaks at $\sim 400 \mathrm{~nm}$. Large nanotubes have an extra peak in PL spectrum. This research is supported by NSF (EAR02-1082) and DOE through LDRD program of Sandia National Laboratories. 


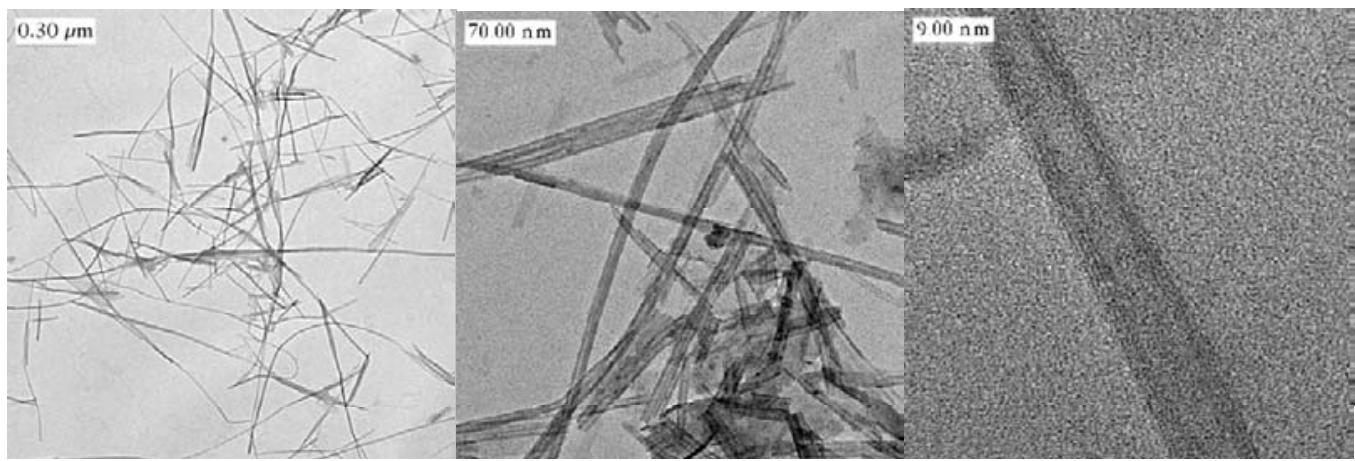

Figure 1: TEM images of the synthesized nanotubes.

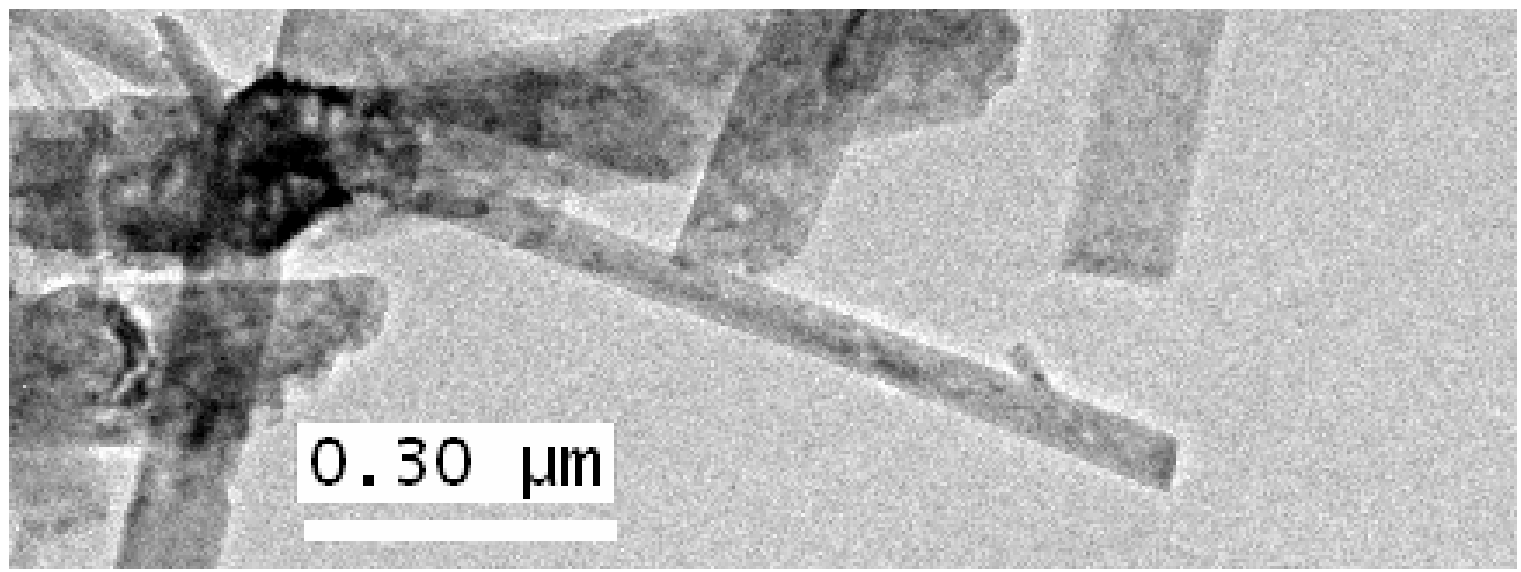

Figure 2: SEM image of the titanate nanofibers.

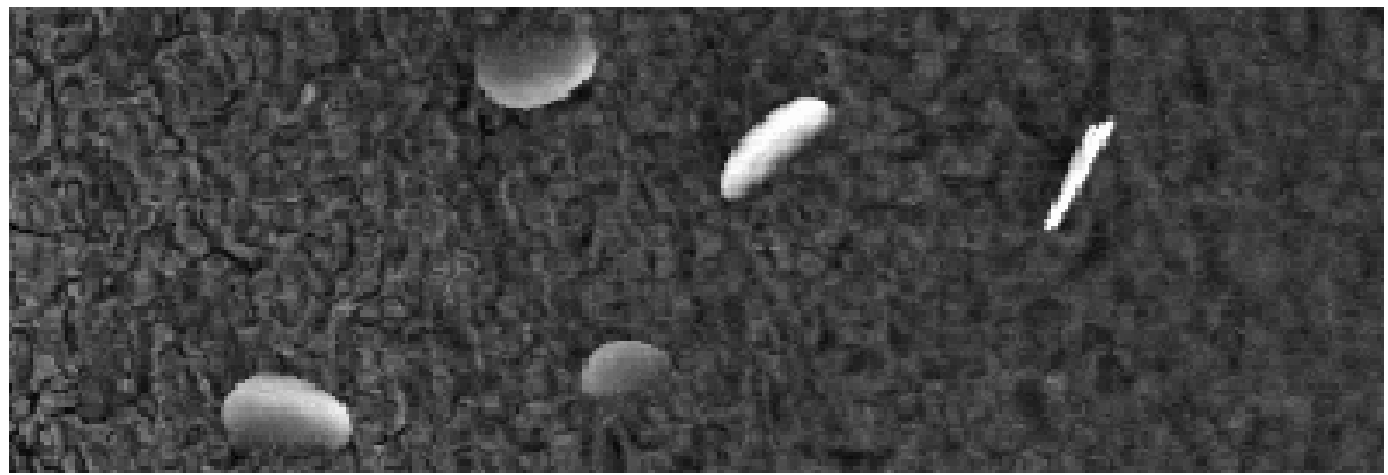

Figure 3: SEM image of titanate nanodiscs. The diameter of the disc is about 2 micron.

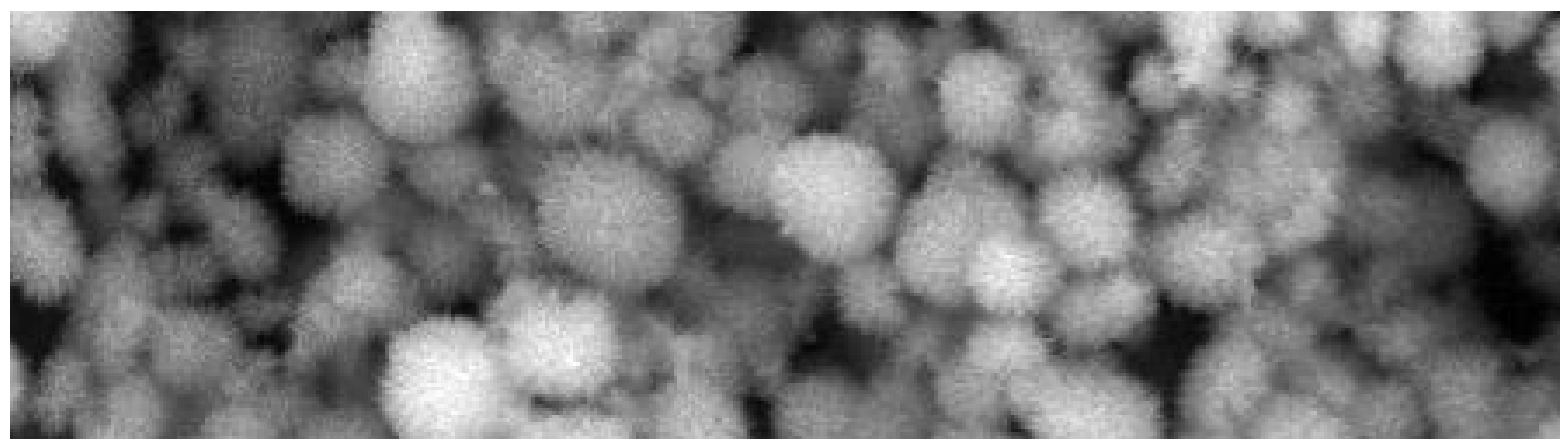

Figure 4: SEM image of titanate nanoflowers. The diameter of the nanoflowers is about 2 microns. 